\title{
GRADATION APPROXIMATION FOR VECTOR BASED COMPRESSION OF COMIC IMAGES
}

\author{
Kei Kawamura $^{\dagger}$, Yuki Yamamoto ${ }^{\dagger}$, and Hiroshi Watanabe ${ }^{\dagger}$ \\ $\dagger$ Graduate School of Global Information and Telecommunication Studies \\ Waseda University \\ 1-3-10, Nishiwaseda, Shinjuku-ku, Tokyo, Japan
}

\begin{abstract}
In this paper, we propose a method to vectorize comic images including halftone dots. Our method can prevent the jaggy and moire phenomena when the images are enlarged and shrank. The method contains three modules: halftone dots separation, gradation approximation, and vectorization. At the first module, small isolated areas and altered areas by dilation and erosion operations are separated into halftone dots images. At the second module, areas of halftone are approximated by contours and gradation parameters. At the last module, both halftone areas and line drawings are vectorized and approximated by smooth contours. The size of compressed file produced by our method is equal or smaller than JBIG compression. Validity of the proposed method is confirmed by experimental results.
\end{abstract}

\section{INTRODUCTION}

The manga, which is Japanese comic, is one of the entertainments of Japanese culture. On one hand large amounts of papers are consumed to distribute weekly comic's magazines. On the other hand, a new display, such as an e-paper and a LCD, is appeared and may replace a paper. To stop expenditure of papers, we design a novel vector based compression of comic images for the new display, which has high resolution such as 300dpi and more. The vectorization data can be transformed into a PostScript file for general application such as draw tool and publication.

A lot of schemes for vectorization or polygon approximation have been proposed so far in the fields of pattern recognition and computer vision [1-4]. Medioni and Yasumoto [1] have presented a method for vectorization consisting of an approximation of a sub-pixel edge by a variable number of Bezier curve segments. Maston and Shih [2] have proposed scale-based dominant point approach that uses a scale-space process to detect dominant one to describe the shape of a 2D object. Yang, Lu, and Lee [3] have presented a curve fitting algorithm for shape description of chinese characters. Kolesnikov and Franti [4] have proposed a fast near-optimal algorithm for min-\# polygonal approximation of digitized curves.

All the above algorithms can be used for the vectorization in comic image compression. However, it is not considered to prevent moire when resolution of an image with halftone dots is changed. In addition, it is redundant to vectorize halftone dots directly. Thus, it is expected that the halftone dots separation provides better results for the image quality and compression rate while a texture of grain on the image is deprived $[5,6]$.

An inverse halftoning problem is to reconstruct a continuous tone image from a halftone dots such that the continuous tone image appears visually similar to the halftone dots. However, the all algorithms in a survey article [7] are considered to produce not a vector graphics but a bitmap images. Thus, we propose a novel tone detection algorithm using adaptive area in the halftone dots for gradation approximation.

Our method consists of three main steps: halftone dots separation, gradation approximation, and vectorization. First we separates input images into line drawings and halftone dots images using two characteristics of halftone dots. Then we detect halftone areas, which are described by contours and gradation parameters. Finally we vectorize, based on polygonal approximation, halftone areas and line drawings. Both of these components are multiplexed together.

\section{OVERVIEW OF VECTOR BASED COMPRESSION METHOD OF COMIC IMAGES}

\subsection{Overview}

A general framework for compression method of comic image, which relies on halftone dots separations, gradation approximations, and Bezier curve approximations of contours, is shown in Fig.1. Input binary images are first separated into line drawings and halftone dots images. Halftone areas are detected by a dilation operation from halftone dots images. A linear gradation tone, including homogeneous one, is represented halftone area in comic images. A grada- 


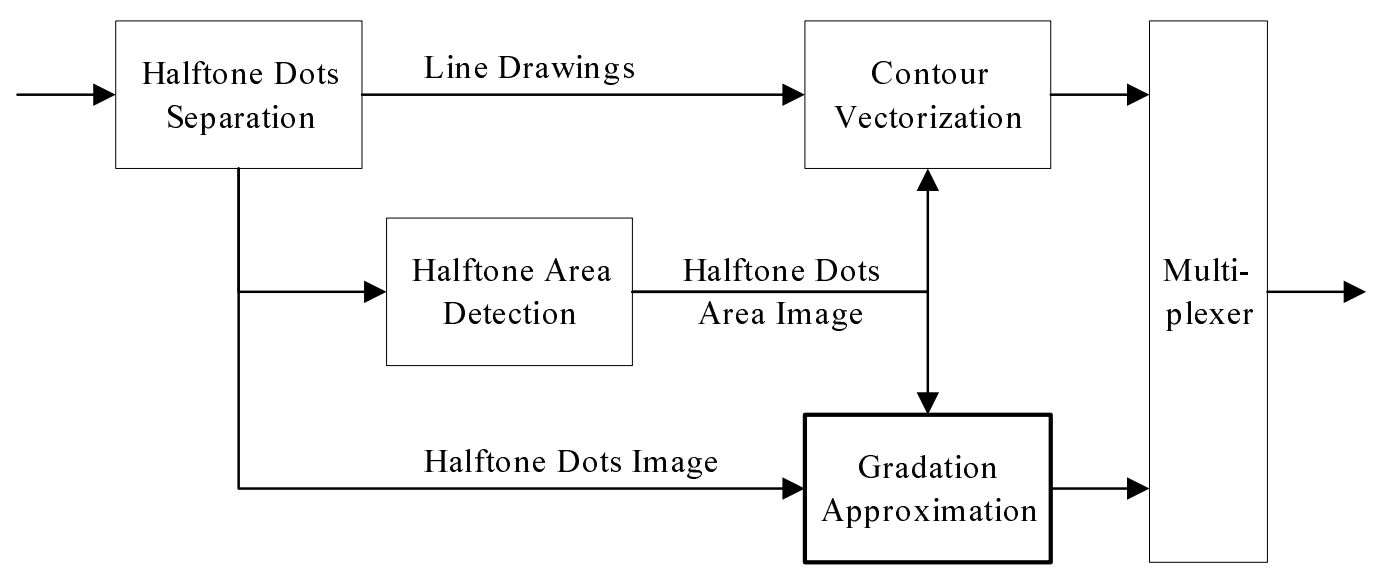

Fig. 1. A block diagram of vector based compression

tion approximation is applied to the each area of halftone. A vectorization based on polygonal approximation of contours is applied to both halftone area images and line drawings. Both of these components are multiplexed together. A tone value is defined as a ratio of halftone dots a unit area.

\subsection{Halftone dots separation}

To obtain halftone dots images, we use two characteristics of them. One is that each dot is an isolated small area where tone value is low or high. The other is that a halftone area would be changed by a dilation operation where tone value is middle.

Halftone areas are detected by a dilation operation from halftone dots images.

\subsection{Vectorization}

We use a conventional algorithm [8] to vectorize a contour of both halftone areas and line drawings. It is based on the polygon approximation for generating a smooth contour made from Bezier curves.

EPS file format, which is produced by the program [8], is redundant with the object of data compression. Thus we use a general text compression program [9] based on BWT (Burrows-Wheeler Transform).

\section{GRADATION APPROXIMATION}

\subsection{Approach}

The main idea behind the proposed method is to approximate a halftone dots by a gradation tone and to vectorize the contour of its area. Since we assume that the original halftone dots represents a homogeneous tone or a linear gradation tone in comic images. We propose to approximate the halftone dots with a smooth contour and some gradation parameters which can be vectorized efficiently.

A linear gradation tone model, which comprehends a homogeneous one, is defined as a plane in 3D space when an image consists of a $\mathrm{x}-\mathrm{y}$ coordinate and a gray level. An equation of the plane, $z=a x+b y+c$, can be solved by the least square method with three or more point's data. Three coefficients (a, b, and c) of the equation are just the gradation parameters. The gray level is defined as a tone value which is calculated by a small area.

\subsection{Constant area size method}

To calculate tone values in the halftone area, it is the simplest way to divide a halftone area into $n$ by $n$ pixels small areas. However, the constant number of $n$ can hardly be used as small area size to calculate tone values because of binary nature of halftone dots.

Since a halftone has a strong period in the density, a moire occurs by a difference of the period of it and the number of $n$. A pattern diagram of above description is shown in Fig.2. When a line of halftone dots is represented in Fig.2 (a), a line of sampling values is represented in Fig.2 (b). A dashed line in Fig.2 (b) is a pseudo tone value. If the number of $n$ is defined as a length of arrows in Fig.2 (c), obtained tone values are dashed lines for each small areas, respectively.

\subsection{Adaptive area size method}

To get tone values without moire, the halftone period should be considered. Technically, it can be obtained easily as follows. We calculate run lengths of black and white pixels, respectively. We obtain distances between the centers of runs. If a series of two distances is same length, there is a periodicity. For example in Fig.2 (d), lengths of arrows 
(a)

(b)

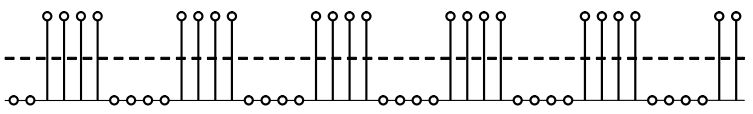

(c)

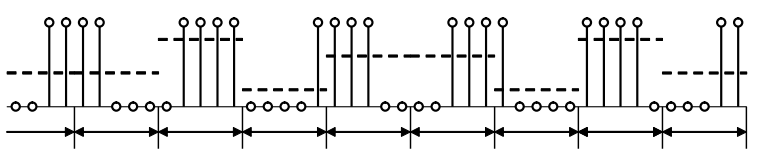

(d)

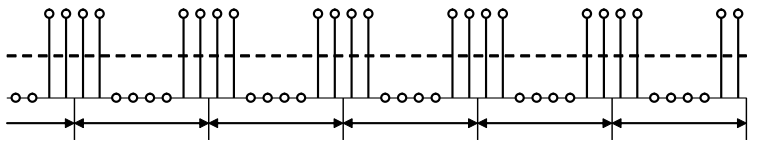

Fig. 2. A pattern diagram of moire occuring

are adaptive number of $n$ and dashed lines are obtained tone values.

\section{EXPERIMENTAL RESULTS}

\subsection{Simulation conditions}

In this section, we demonstrate the validity of halftone dots separation, gradation approximation, and vectorization on some comic images. We compare the obtained gradation parameters with the theoretical one. We also compare our compression rate with the JBIG compression under some conditions.

The test images, which are scanned at the resolution of $300 \mathrm{dpi}$, include three pages with halftone dots. They have 1486x2208 pixels. They are selected from comics.

\subsection{Halftone dots separation and gradation approxima- tion results}

Our halftone dots separation operates on both an input image and an inverse input image, in which black and white pixels are inverted. Fig. 3 shows a part of an input image.

The halftone dots separation achieved with the proposed method is illustrated in Fig.4, Fig.5, and Fig.6. The validity of the gradation approximation can be seen in Fig.7 and Fig.8.

\subsection{Gradation parameter detection results}

We evaluate the approximation method using following process. First, we generate gradation images using continuous tone in computer. Next, we convert them to binary image using halftone dots method. Then, the proposed method is applied to them and gradation parameters are obtained.

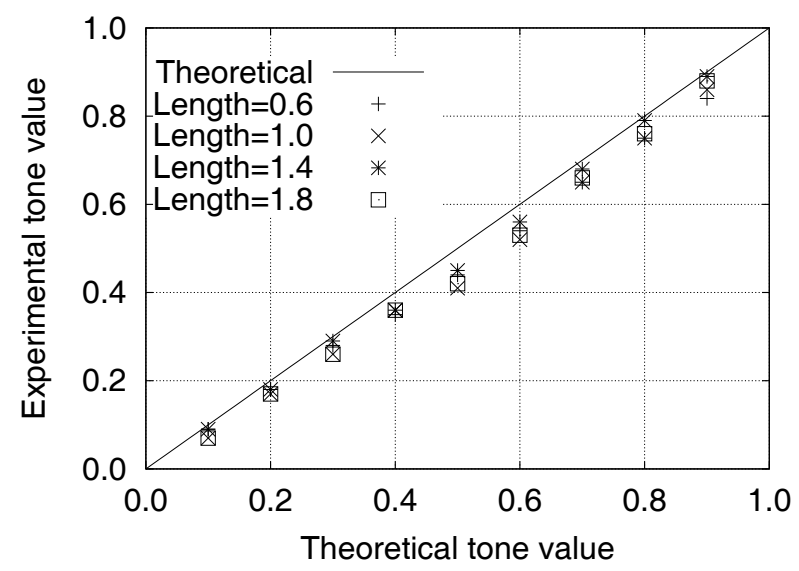

Fig. 9. A relation of gradation length and max density

Table 1. Comprassed file size [Byte]

\begin{tabular}{c|r|r|r}
\hline No. & JBIG & Simple Vector & Proposed Vector \\
\hline 1 & 67016 & 69639 & 53358 \\
\hline 2 & 52704 & 60251 & 58762 \\
\hline 3 & 50511 & 44137 & 30231 \\
\hline
\end{tabular}

Finally, we compare known gradation parameters and obtained ones. Gradation parameters are transformed to lowest density, gradation direction, and slop.

The cumulative results are shown for the theoretical line and four lengths of gradations in Fig.9. The theoretical tone values are plotted versus the experimental ones. This figure shows that the proposed adaptive small area method reconstructs the theoretical tone values.

\subsection{Compression rate}

The size of compressed file of JBIG and Vectors are shown in Table1, in which a simple vector is defined as only vectorized image without halftone dots separation.

Since no. 2 test image contains halftone dots plus another texture, the size of proposed vector is larger than the JBIG but smaller than the simple vector. The other cases, proposed vector are the smallest size. The validity of the proposed method is confirmed by this table.

\section{CONCLUSIONS}

In this paper, we proposed a gradation approximation for vector based image compression method of binary comic images. It consisted of three main steps: halftone dots separation, gradation approximation, and vectorization. Images were first separated into line drawings and halftone dots im- 


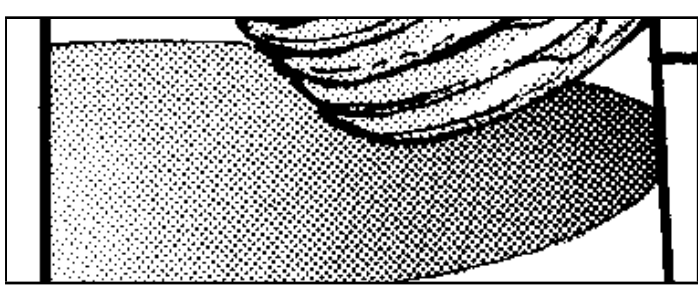

Fig. 3. Input image

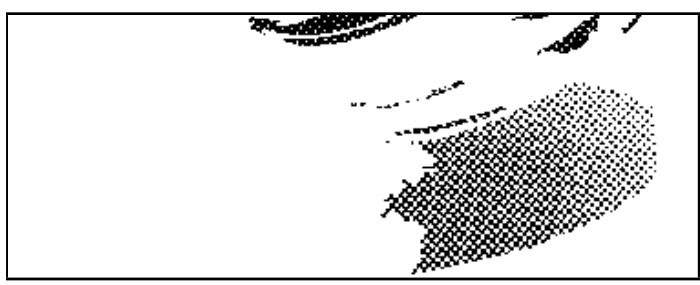

Fig. 5. Inverce halftone-dots image at high density

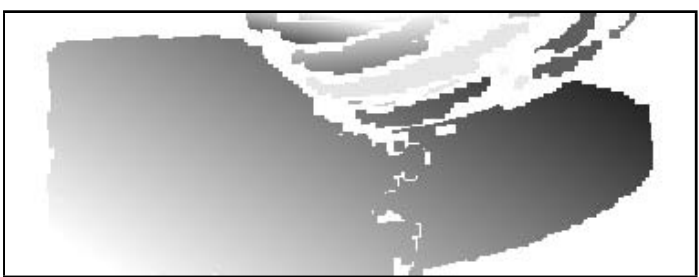

Fig. 7. Gradation approximation image

ages using two characteristics of halftone dots. Each area of a halftone was described by contours and gradation parameters. A vectorization based on polygonal approximation was applied to halftone areas and line drawings. Both of these components were multiplexed together. Validity of the proposed method was confirmed by experimental results.

\section{REFERENCES}

[1] G. Medioni, Y. Yasumoto, "Corner detection and curve representation using cubic B-splines," IEEE Int. Conf. on Robotics and Automation 1986, Volume 3, pp. 764769, Apr. 1986.

[2] R.E. Marston, J.C. Shih, "Polygonal approximation of outlines by scale-based dominant point detection," Fifth Int. Conf. on Image Processing and its Applications 1995, pp. 350-354, Jul. 1995.

[3] Hsi-Ming Yang, Jainn-Jyh Lu, Hsi-Jian Lee, "A Bezier curve-based approach to shape description for Chinese calligraphy characters," Sixth Int. Conf. on Document Analysis and Recognition 2001, pp. 276-280, Sept. 2001.

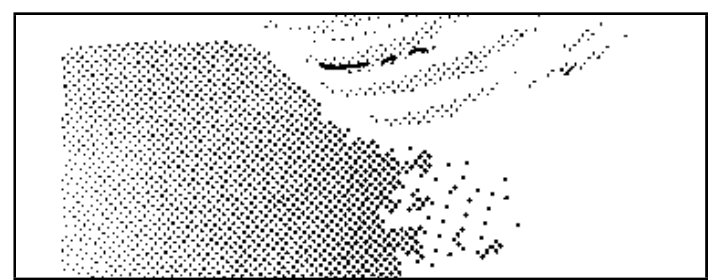

Fig. 4. Halftone-dots image at low density

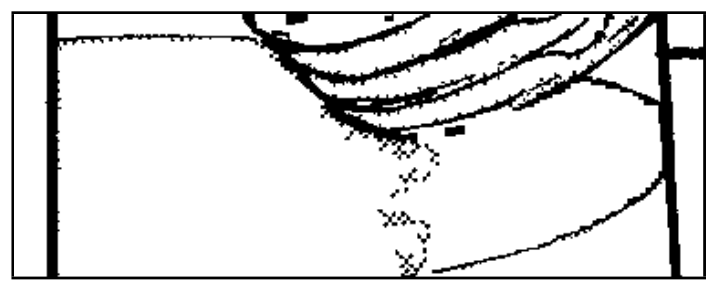

Fig. 6. Line drawing

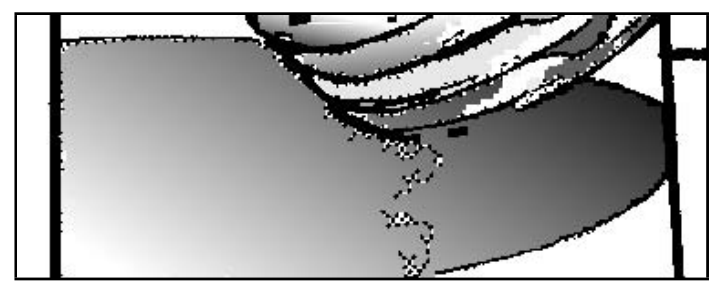

Fig. 8. Reconstructed image

[4] A. Kolesnikov, P. Franti, "A fast near-optimal algorithm for approximation of polygonal curves," 16th Int. Conf. on Pattern Recognition 2002, Volume. 4, pp. 335-338, 2002.

[5] O. Nakagami, T. Miyazawa, H. Watanabe, H. Tominaga, "A Study on two-layer coding for animation images," IEEE Int. Conf. on Multimedia and Expo (ICME) 2002, WedAmPO3: Compression II, Aug. 2002.

[6] K. Kawamura, H. Watanabe, H. Tominaga, "Vector Representation of Binary Images Containing Halftone Dots," IEEE Int. Conf. on Multimedia and Expo (ICME) 2004, TP2-1, Jun. 2004.

[7] P. W. Wong, N. Memon, "Image processing for halftones," IEEE Signal Processing Magazine, Vol.20, No.4, pp.59-70, Jul. 2003.

[8] Peter Selinger, "Potrace: a polygon-based tracing algorithm," http://potrace.sourceforge.net/ potrace.pdf, 2003.

[9] Damien Debin, “zzip," http://debin.org/ zzip/, 2001. 